\title{
Correcting Observation Errors for Assembly Task Recognition
}

\author{
Jun Takamatsu Koichi Ogawara Hiroshi Kimura† Katsushi Ikeuchi \\ Institute of Industrial Science \\ $\dagger$ Graduate School of Information Systems \\ The University of Tokyo \\ Tokyo, 106-8558, Japan \\ University of Electro-Communications \\ Tokyo, 182-8585, Japan
}

\begin{abstract}
The completion of robot programs requires long development time and much effort. To shorten the programming time and to minimize the effort, we have been developing a system which we refer to as the "assembly-plan-from-observation (APO) system."

This system requires assembly task recognition from observing human performance. Observation data by a robot's vision system is usually error contaminated, and, thus, we cannot use those data directly. This paper proposes two methods to clean up those errors by using contact relations and their transitions. The first one corrects the observed configuration from contact relations observed. The second one identifies wrongly determined contact relations from an analysis of configuration space ( $C$-space). We have implemented both methods on our test bed and have verified their effectiveness.
\end{abstract}

\section{Introduction}

The completion of robot programs requires long development time and much effort. To shorten the programming time and to minimize the effort, we have been developing the assembly plan from observation (APO) system[1]. The goal of the APO system is enable us to design and develop a robot that can perform assembly tasks by observing how humans perform those tasks.

The APO system requires precise configuration of objects for recognizing assembly tasks. Unfortunately, that obtained data usually contains errors. Those errors should be removed before utilizing the data in the system. Xiao and Zhang proposed a method to solve the contact formulation analytically in [5]. To our knowledge, they only developed a method to solve planer cases. Suehiro and Ikeuchi proposed a method to correct the errors using face contact relations[2]. Their method can handle only face-to-face relations; it cannot handle general relations, including face-to-vertex and edge-to-edge relations. We propose a method to determine precise object configurations using observed contact relations.
In this paper, we also propose a method to identify wrongly determined contact relations. A correct contact relation is necessary for the method mentioned above. Although a contact relation is usually robust to vision errors, sometimes wrong contact relations are obtained. In this paper, we propose a method to identify these wrongly determined contact relations by using an analysis of the configuration space (C-space).

Section 2 describes various concepts necessary to explain later sections. Section 3 describes a method to calculate the correct object configuration based on a contact relation. Section 4 develops a method to remove wrongly determined contact relations based on the C-space analysis. Section 5 explains the experimental result. Section 6 presents our conclusions.

\section{Preliminaries}

In this section, we describe various concepts necessary to introduce our proposed method. First, we describe contact relations that are indispensable for analyzing assembly tasks. Next, we describe a motion degree of freedom (DOF) that are useful for identifying wrongly determined contact relations (See in Section 4).

This paper concentrates on the correct relative configuration between two objects. We consider that general n-object relations can be corrected as a series of these two-object corrections. We also assume that all objects are rigid and polyhedral.

\subsection{Contact relation}

A contact relation consists of contact-element relations. A polyhedral object is composed of vertices, edges and faces. These are contact elements. Contact-element relations are defined as those between contact elements. A contact relation can be represented as a combination of contact-element relations.

Three contact-element relations are the most important ones. Contact-element relations can be classified into nine types (Shown in Figure 1). How- 


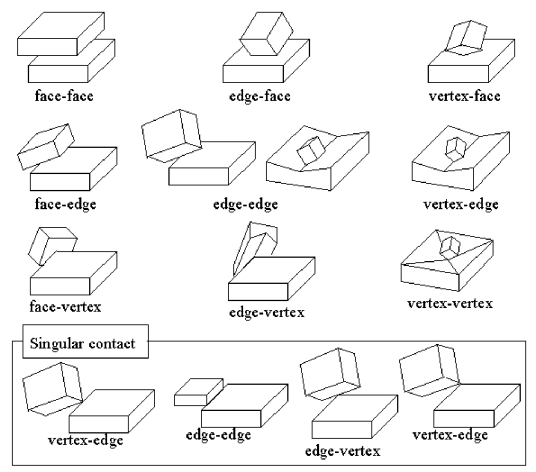

Figure 1: Nine types of contact elements

ever, by considering possible local displacements, three contact elements, vertex-face, face-vertex, and non-parallel edge-edge contacts, are enough; other contact-element relations can be represented as a combination of these three contact-element relations. ${ }^{1}$ For example, possible local displacements under an edge-face contact as shown in Figure 2 (a) are equivalent to those under two vertex-face contacts as shown in Figure 2 (b).

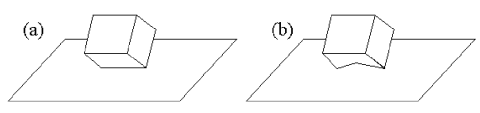

Figure 2: Edge-face contact

\section{$2.2 \quad$ Motion DOFs}

For recognizing assembly tasks, we define three types of motion DOFs in translation and rotation[3] (Shown in Figure 3);

Maintaining DOF An object can move while still maintaining the contact relation in the DOF.

Detaching DOF An object can move with breaking the contact relation in the DOF.

Constraining DOF An object cannot move in the DOF.

\section{Calculating correct object configura- tions based on contact relations}

This section describes a method to determine correct object configurations based on contact relations. First, we determine contact relations from erroneously obtained object configurations. Then, using the obtained contact relations, we calculate correct object configurations

\footnotetext{
${ }^{1}$ In this paper, an edge-edge contact means a non-parallel edge-edge contact.
}

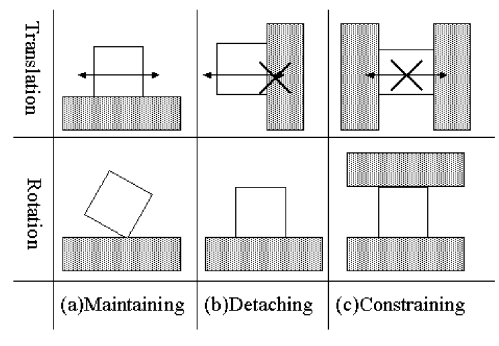

Figure 3: Motion DOFs

\subsection{Determining contact relations from con- taminated observation}

In order to determine contact relations under contaminated data, we employ a two-step procedure. The first step chooses a candidate set of contactelement relations using distances between contact elements, e.g., vertices, edges, and faces. When the distance is less than a certain threshold, we consider that those elements are in contact with each other.

The second step determines a contact relation by using all possible candidates of contact-element relations. If two objects penetrate each other near a certain candidate, we remove this candidate from the candidate set. The resulting set is considered to be the contact relation.

The following paragraphs explain the second step, cutting down on possible candidates, in each contactelement relation.

Vertex-face, face-vertex contact: Consider the case of a vertex-face contact as shown in Figure 4. The candidate is removed from the set, if some of the edges connecting to the vertex penetrate the face. Namely, it must be satisfied that $\exists i, n \cdot e_{i}<0$, where $n$ is the face normal and $e_{i}$ is the direction of an i-th edge. Due to observation errors, the contact candidate is removed, if $\frac{\left|n \cdot e_{i}\right|}{n \cdot e_{i}}$ is less than a certain threshold. We can apply the same argument to a face-vertex contact.
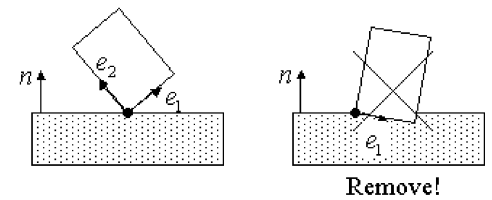

Figure 4: Two types of vertex-face contact candidates

Edge-edge contact: Consider the case of an edgeedge contact as shown in Figure 5. The candidate is removed if one edge $e_{1}$ penetrates some of the connecting faces to the other edge $e_{2}$. Here, $n_{1 p}$ and $n_{1 n}$ are normals of faces connecting to edge $e_{1}, n_{2 p}$ and $n_{2 n}$ are normals of faces connecting to edge $e_{2}$, 
$l_{1}$ and $l_{2}$ are directions of edges $e_{1}$ and $e_{2}$, and ang is an appropriate threshold.

1. Return with the contact relation if $\left(n_{1 p} \cdot l_{2}\right)\left(n_{1 n}\right.$. $\left.l_{2}\right)<0$.

2. If $\pi / 2-\cos ^{-}\left(n_{1 p} \cdot l_{2}\right)<a n g$, go to step 3. Else, go to step 4.

3 . Return with the contact relation if $\pi / 2-$ $\cos ^{-}\left(n_{1 p} \cdot n_{2 p}\right)<a n g$ and $\pi / 2-\cos ^{-}\left(n_{1 p} \cdot n_{2 p}\right)<$ ang

4. If $\pi / 2-\cos ^{-}\left(n_{1 n} \cdot l_{2}\right)<a n g$, go to step 5. Else go to step 6.

5 . Return with the contact relation if $\pi / 2-$ $\cos ^{-}\left(n_{1 n} \cdot n_{2 p}\right)<$ ang and $\pi / 2-\cos ^{-}\left(n_{1 n} \cdot n_{2 p}\right)<$ ang.

6. Remove this candidate.

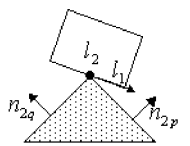

[correspond to 1.]

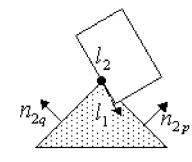

[correspond to 2. and 4.]

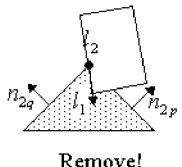

Remove!
Figure 5: Three types of edge-edge contact candidates

\subsection{Calculating a correct object configura- tion using a contact relation}

Generally speaking, calculating the object configuration with the given contact relation requires solving simultaneous non-linear redundant equations. In this paper, we will employ the iterative least square method for solving these equations. This iterative process needs an initial approximation. For the initial values, we use those obtained from observation[2]. The method is as follows:

1. Set up a contact equation for each contactelement relation.

2. Transform these equations to linear ones using the Taylor expansion.

3. Solve the simultaneous linear equations using the least square method.

4. Repeat Steps 1 to 3 until the solution converges.

Setting up a contact equation. The object configuration obtained from observation includes some errors. We will correct these errors by moving the object a little. When the object rotates $\gamma, \beta$, and $\alpha$ around z-axis, $\mathrm{y}$-axis, and $\mathrm{x}$-axis, and translates $x$, $y, z$ along $\mathrm{x}$-axis, $\mathrm{y}$-axis, and $\mathrm{z}$-axis, a surface normal $n$ and a vertex $v$ change to $n^{\prime}$ and $v^{\prime}$ as shown in equation (1), where $R_{x}(\theta), R_{y}(\theta)$, and $R_{z}(\theta)$ are the $3 \times 3$ matrices representing the rotation $\theta$ around $\mathrm{x}$-axis, $\mathrm{y}$-axis, and z-axis.

$$
\begin{gathered}
n^{\prime}=R n \\
v^{\prime}=R v+T \\
R=R_{x}(\alpha) R_{y}(\beta) R_{z}(\gamma) T=(x y z)^{T}
\end{gathered}
$$

Vertex-face contact: Consider the vertex-face contact case as shown in Figure 6. Because the vertex $v^{\prime}$ must be on the face $f$ after error correction, equation (2) is obtained.

$$
\begin{aligned}
\Delta e_{v f} & =n \cdot\left(v^{\prime}-f\right) \\
& =n \cdot(R v+T-f)=0
\end{aligned}
$$

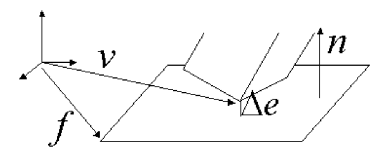

$\begin{aligned} & \text { Figure 6: Vertex-face } \begin{array}{l}\text { Figure } \\ \text { contact }\end{array} \\ & \text { contact }\end{aligned}$

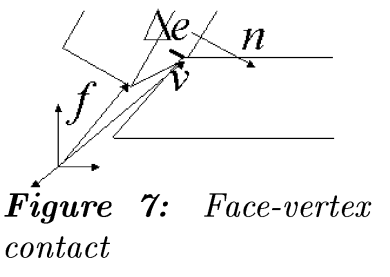

Face-vertex contact: Consider the face-vertex contact case as shown in Figure 7. After error correction, a vertex $f^{\prime}$ on the face and a face normal $n^{\prime}$ are calculated using equation (3).

$$
\begin{aligned}
& n^{\prime}=R n \\
& f^{\prime}=R f+T
\end{aligned}
$$

Because the vertex $v$ must be on the face $f^{\prime}$, equation (4) is obtained.

$$
\begin{aligned}
\Delta e_{f v}= & n^{\prime} \cdot\left(v-f^{\prime}\right) \\
& =R n \cdot(v-R f-T) \\
& =R n \cdot v-n \cdot f-R n \cdot T=0 \\
& \quad\left(\because R^{T} R=E\right)
\end{aligned}
$$

Edge-edge contact: Consider the edge-edge contact case as shown in Figure 8. After error correction, a direction vector $e_{1}^{\prime}$ and a point $p_{1}^{\prime}$ on the edge are computed in equation (5).

$$
\begin{aligned}
& e_{1}^{\prime}=R e_{1} \\
& p_{1}^{\prime}=R p_{1}+T
\end{aligned}
$$

Because the edge $E_{1}$ must be in contact with the edge $E_{2}$, equation (6) is obtained.

$$
\begin{aligned}
\Delta e_{e e} & =\frac{\left(e_{1}^{\prime} \times e_{2}\right) \cdot\left(p_{2}-p_{1}^{\prime}\right)}{\left|e_{1}^{\prime} \times e_{2}\right|} \\
& =\frac{\left(R e_{1} \times e_{2}\right) \cdot\left(p_{2}-R p_{1}-T\right)}{\left|R e_{1} \times e_{2}\right|}=0
\end{aligned}
$$




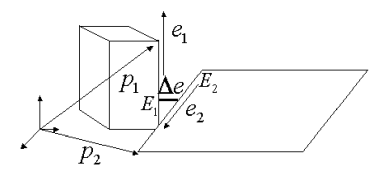

Figure 8: Edge-edge contact

Taylor Expansion. Applying the Taylor Expansion near the present object configuration to equations (2), (4), and (6), equations (7), (8), and (9) are obtained, where $r=(\alpha, \beta, \gamma)^{T}$ and $P=$ $\frac{p_{2}-p_{1}+\left\{n \cdot\left(p_{2}-p_{1}\right)\right\} n}{\left|e_{1} \times e_{2}\right|}$.

$$
\begin{aligned}
\Delta e_{v f} & =n \cdot(v-f)-\left[-n^{T}(n \times v)^{T}\right]\left[\begin{array}{l}
T \\
r
\end{array}\right](7) \\
\Delta e_{f v} & =n \cdot(v-f)-\left[n^{T}(v \times n)^{T}\right]\left[\begin{array}{l}
T \\
r
\end{array}\right] \\
\Delta e_{e e} & =n \cdot\left(p_{2}-p_{1}\right) \\
& -\left[n^{T}\left\{p_{1} \times n+\left(e_{2} \times P\right) \times e_{1}\right\}^{T}\right]\left[\begin{array}{l}
T \\
r
\end{array}\right] 9
\end{aligned}
$$

All of the equations can be represented in the form as shown in the equation (10).

$$
\Delta e=c-\left[a^{T} b^{T}\right]\left[\begin{array}{l}
T \\
r
\end{array}\right]
$$

Least square method. For the least square method, we solve the simultaneous equations (11), where $q=\left[\begin{array}{l}T \\ r\end{array}\right]$.

$$
\frac{\partial}{\partial q} \sum(\Delta e)^{2}=0
$$

By expanding equation (11), equation (12) is obtained, where $\mathrm{n}$ is the number of contact elements.

$$
\begin{aligned}
& \frac{\partial}{\partial q} \sum(\Delta e)^{2}\left.=\sum_{i}^{n} \frac{\partial}{\partial q}\left(c_{i}-\left[\begin{array}{ll}
a_{i}^{T} & b_{i}^{T}
\end{array}\right] q\right)^{2}\right) \\
&=\sum_{i}^{n}\left[\begin{array}{c}
a_{i} \\
b_{i}
\end{array}\right]\left(c_{i}-\left[\begin{array}{ll}
a_{i}^{T} & b_{i}^{T}
\end{array}\right] q\right)=0 \\
& {\left[\sum_{i}^{n}\left(\begin{array}{c}
a_{i} \\
b_{i}
\end{array}\right)\left[\begin{array}{ll}
a_{i}^{T} & b_{i}^{T}
\end{array}\right]\right] q=\sum_{i}^{n} c_{i}\left[\begin{array}{c}
a_{i} \\
b_{i}
\end{array}\right] }
\end{aligned}
$$

Because the rank of the matrix $\sum_{i}^{n}\left[\begin{array}{c}a_{i} \\ b_{i}\end{array}\right]\left[\begin{array}{ll}a_{i}^{T} & b_{i}^{T}\end{array}\right]$ may not be six, we solve the simultaneous equations by using the singular value decomposition method.

\section{Identifying wrongly determined con- tact relations}

The previous section assumes that the obtained contact relations are correct. However, under a large observation error, some of the contact relations may not be obtained correctly. In this section, we propose a method to identify those contact relations based on an analysis in $\mathrm{C}$-space. More precisely, we will examine possible direct transitions in $\mathrm{C}$-space. ${ }^{2}$

First, we consider the relationship between possible direct transitions and connections between Csurfaces in C-space[7]. Here, C-surface is defined as the surface in C-space corresponding to a set of possible object configurations that maintain one particular contact relation. Note that an edge or a vertex between two $\mathrm{C}$-surfaces is a different C-surface from them.

Next, we propose a fast, practical algorithm for determining possible direct transitions.

\subsection{Possible transitions and C-surface}

Consider the transitions between contact relations as shown to the left in Figure 9. The arrows represent possible direct transitions. C-surfaces of corresponding contact relations are shown to the right in Figure 9 in C-space.

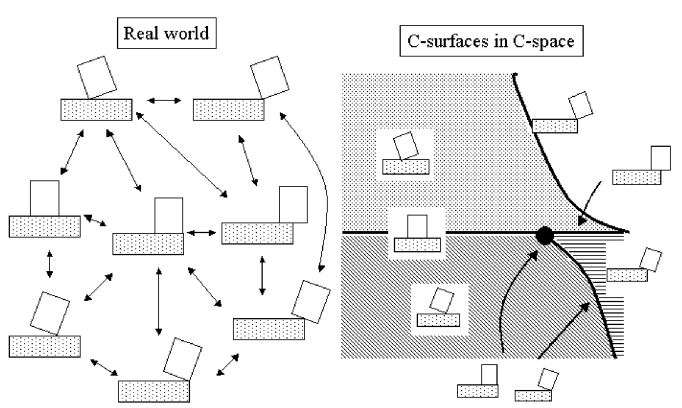

Figure 9: Relationship between possible transitions and $C$-surface

The possible direct transition between two contact relations $A$ and $B$ exists if, and only if,

$$
\begin{gathered}
\left\{a_{i}\right\} \in \mathrm{C} \text {-surface of } A \text { and } b \in \mathrm{C} \text {-surface of } B, \\
\qquad \lim _{i \rightarrow \infty}\left\{a_{i}\right\}=b \text { or } \\
\left\{b_{i}\right\} \in \mathrm{C} \text {-surface of } B \text { and } a \in \mathrm{C} \text {-surface of } A, \\
\lim _{i \rightarrow \infty}\left\{b_{i}\right\}=a,
\end{gathered}
$$

in short, C-surfaces of $A$ and $B$ are adjacent to each other.

\subsection{Determining possible direct transitions}

As mentioned above, we can decide whether the transition is possible based on the connection between

\footnotetext{
${ }^{2} \mathrm{~A}$ possible direct transition means that one contact relation can transit to another without any intermediate contact relation.
} 
two C-surfaces. However, it is very difficult to calculate all connections among all the $\mathrm{C}$-surfaces given by two polyhedra. Fortunately, since we have already obtained contact relations from observation, we only need to construct a local C-space using observed contact relations to determine whether obtained transitions are possible.

Before introducing the proposed method, we examine the characteristics of $\mathrm{C}$-surfaces:

- One contact relation corresponds to one, and only one, unique $\mathrm{C}$-surface.

- One configuration corresponds to one, and only one, unique C-surface.

- A set composed of the C-surfaces that contain a certain contact-element relation is a closed set.

Next, we prove some propositions:

Proposition 1 Given two contact relations $A$ and $B$, the corresponding $C$-surfaces $C_{a}$ and $C_{b}$ are not connected directly, if $\exists a \in A, a \notin B$ and $\exists b \in B, b \notin$ A

( $a$ and $b$ are contact elements. $)^{3}$

Proof Assuming C-surfaces $C_{a}$ and $C_{b}$ are connected, it must be satisfied that $\left\{a_{i}\right\} \in C_{a}$ and $b \in C_{b}, \lim _{i \rightarrow \infty}\left\{a_{i}\right\}=b$, or $\left\{b_{i}\right\} \in C_{b}$ and $a \in C_{a}$, $\lim _{i \rightarrow \infty}\left\{b_{i}\right\}=a$. Here, one may consider that the former is satisfied. Considering a certain contactelement relation $p$ satisfying $\exists p \in A, p \notin B$. The contact-element relation $p$ is detached while the transition from $C_{a}$ to $C_{b}$. But the set composed of Csurfaces that contain the contact element $p$ is a closed set. Since there is a set that approaches to $b$ infinitely, the $b$ should be inside of the closed set; it must be satisfied that $b \in C_{a}$. That is contrary to the fact that one configuration, $b$ corresponds to one, and only one, C-surface.

Proposition 2 Given two contact relations $A$ and $B$, the corresponding $C$-surface $C_{a}$ and $C_{b}$ are not connected directly if constraining DOFs in one contact relation translate maintaining or detaching DOFs in the other contact relation.

Proof Shown in Figure 10. In that case, the entrance contact relation exists between these transitions. Therefore, these transitions are impossible. For more detailed proof, see [3]

\footnotetext{
${ }^{3}$ In this paper, a parallel edge-edge contact is represented as the combination of several vertex-face and face-vertex contacts. A parallel edge-edge contact needs to be represented as the combination added to the edge-edge contact, when applying the method
}

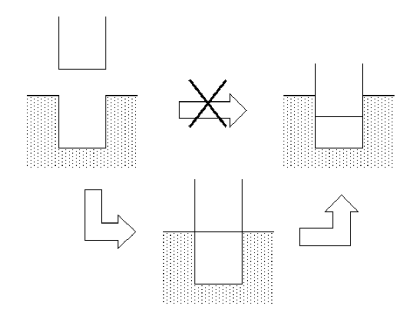

Figure 10: Impossible direct transition

From Proposition 1 and 2, the algorithm for deciding possible direct transitions is as follows:

Given two contact relations $A$ and $B$,

- The transition is impossible if $\exists a \in A, a \notin B$ and $\exists b \in B, b \notin A$.

- The transition is impossible if constraining DOFs in one contact relation translate maintaining or detaching DOFs in the other contact relation.

- Else, the transition is possible.

\subsection{Example of identifying wrongly transla- tions}

Considering four contact relations as shown in Figure 11: all contact-element relations of the right contact relation are included by contact-element relations of the middle contact relation, so a transition between these two relations is possible; all contact-element relations of the upper left contact relation are included by contact-element relations of the right contact relation. In this case, a maintaining DOF transits to a constraining DOF directly: thus, a transition between these two relations is impossible.

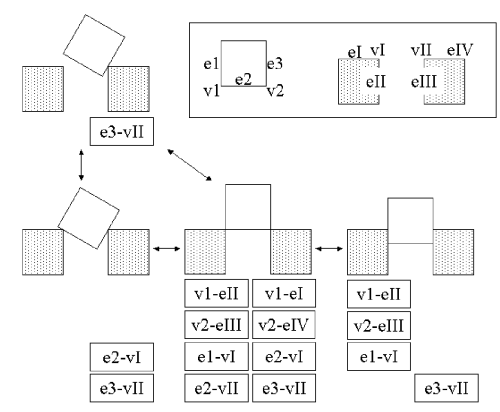

Figure 11: Example: possible direct transitions

\section{Experiments}

We verified our proposed method using a test bed "Dot-chan" [4] as shown in Figure 12. First, we performed the peg-in-hole assembly task in front of the 


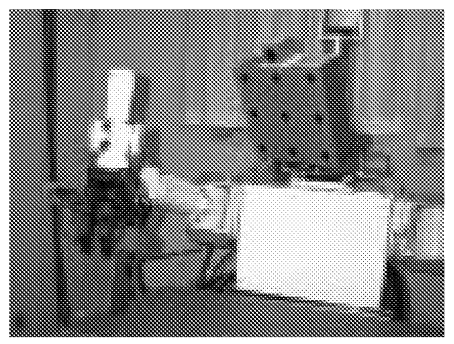

Figure 12: A test bed "Dot-chan"

multi-baseline real time stereo system[8] on the Dotchan. It obtained 2D and 3D images. By applying background subtraction and 3DTM[6] to these images, trajectories of two objects were obtained.

After removing vision errors, the robot obtained correct trajectories and correct direct transitions (Shown in Figure 13 and 14).

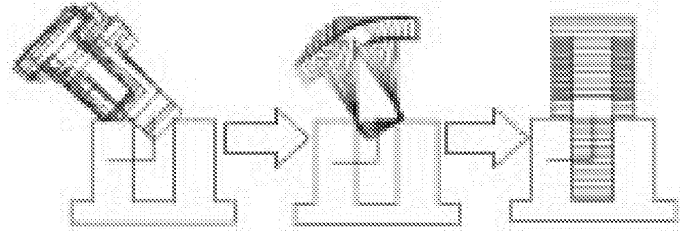

Figure 13: Correcting observation errors

\section{Conclusions}

We have proposed the method for robustly correcting observation errors by using contact relations that are assumed to have been correctly obtained. We also proposed a method to identify and remove poorlydetermined contact relations by using an analysis in the C-space. Here we employ two propositions given by the characteristics of C-space. By using these two methods, the robot can acquire the correct object configurations that are necessary to perform robot

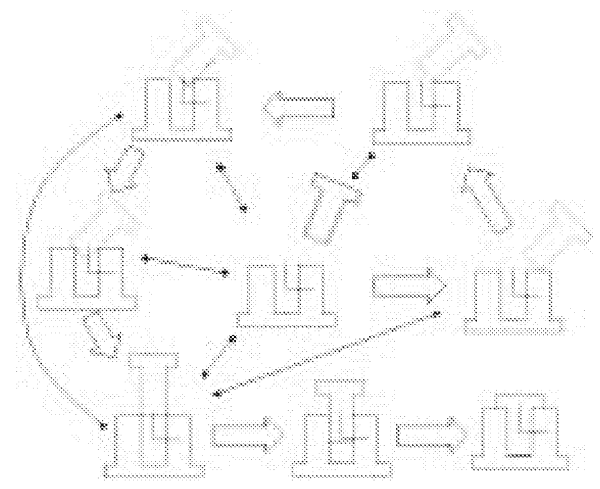

Figure 14: Possible direct transitions actions from observing human demonstrations.

\section{Acknowledgments}

This work is supported in part by the Japan Science and Technology Corporation (JST) under the Ikeuchi CREST project, and in part by the Grant-in-Aid for Scientific Research on Priority Areas (C) 13224025 of the Ministry of Education, Culture, Sports, Science and Technology.

\section{References}

[1] K. Ikeuchi and Takashi Suehiro, "Toward an Assembly Plan from Observation Part I: Task Recognition with Polyhedral Objects", IEEE Transactions on Robotics and Automation, Vol 10, No. 3, June, 1994.

[2] Takashi Suehiro and Katsushi Ikeuchi, "Towards an Assembly Plan from Observation: Part II: Correction of Motion Parameters based on Fact Contact Constraints", IEEE Int. Conf. on Intelligent Robots and Systems, pp. 2095 - 2102, July 7 - 10, 1992.

[3] Jun Takamatsu, Hirohisa Tominaga, Koichi Ogawara, Hiroshi Kimura, and Katsushi Ikeuchi, "Extracting Manipulation Skills from Observation", IEEE Int. Conf. on Intelligent Robots and Systems, Vol. 1, pp. 584 - 589, 2000.

[4] K. Ogawara, J. Takamatsu, S. Iba, T. Tanuki, Y. Sato, A. Saegusa, H. Kimura, and K. Ikeuchi, "Acquiring hand-action models in task and behavior levels by a learning robot through observing human demonstrations", The First IEEERAS International Conference on Humaniod Robots, 2000.

[5] J. Xiao and L. Zhang, "Contact Constraint Analysis and Determination of Geometrically Valid Contact Formations from Possible Contact Primitives," IEEE Trans. on Robotics and Automation, Vol.13, No.3, pp. 456-466, June 1997.

[6] Mark. D. Wheeler and K. Ikeuchi, "Sensor Modeling, Probabilistic Hypothesis Generation, and Robust Localization for Object Recognition", IEEE Trans. on Pattern Analysis and Machine Intelligence, Vol. 28, pp 293 - 331, 1986.

[7] T. Lozano-Perez, M. T. Mason, and R. H. Taylor, "Automatic Synthesis of Fine-Motion Strategies for Robtics", IEEE International Journal of Robotics Research, Vol. 3, No. 1, pp. 3 - 24, 1984.

[8] S. Kimura, T. Shinbo, E. Kawamura, H. Yamaguchi, and K. Nakano, "A New Real-Time Stereo Vision System Using 2D Convolvers", Technical Report of IEICE, Vol. 97, No. 501, PRMU97-207, pp. 1- 8, January, 1998. 